\title{
Vajilla broncínea tardorrepublicana en El Llano de la Horca (Santorcaz, Madrid)
}

\section{Late Roman Republican bronze vessels in El Llano de la Horca (Santorcaz, Madrid)}

\author{
Sandra Azcárraga Cámara \\ Enrique Baquedano Pérez \\ Gabriela Märtens Alfaro \\ Miguel Contreras Martínez \\ Museo Arqueológico de la Comunidad de Madrid \\ Gonzalo Ruiz Zapatero \\ Universidad Complutense de Madrid
}

\section{RESUMEN}

El reciente avance en la investigación de yacimientos de la Segunda Edad del Hierro del interior peninsular está aportando datos reveladores sobre diversos materiales de cronología tardorrepublicana y procedencia itálica. El hallazgo en El Llano de la Horca de diversos elementos de la vajilla de bronce de esta época y origen, supone la mayor concentración de este tipo de materiales en el entorno y añade importante información acerca de la presencia de elementos importados en la Submeseta Sur.

\section{SUMMARY}

Recent progress made researching Second Iron Age sites in central Iberian Peninsula shows revealing data on different Late Roman Republic materials from Italy. The discovery of several bronze vessels at El Llano de la Horca might contribute important information regarding the ongoing research on imported materials in the Southern Submeseta.

PALABRAS CLAVES: Vajilla de bronce tardorrepublicana, El Llano de la Horca, objetos importados.

KEY WORDS: Bronze vessels, Late Roman Republican period, El Llano de la Horca, imported materials.

\section{INTRODUCCIÓN}

La vajilla de barniz negro en gran medida y los contenedores anfóricos, en menor cantidad, han focalizado hasta el momento el estudio de las importaciones itálicas tardorrepublicanas en el centro de la Península Ibérica. Conviene recordar que dicho territorio se ha considerado tradicionalmente poco receptivo a los procesos de cambio iniciados con la romanización en momentos relativamente tempranos. Lo cierto es, que hasta fechas recientes, los materiales de cronología tardorrepublicana eran prácticamente inexistentes en la zona central de la Península. Este vacío venía impuesto, a nuestro juicio, por la falta de excavaciones en extensión y sobre todo la escasa publicación de los datos. En los últimos años estos materiales están saliendo a la luz, principalmente el barniz negro itálico, en diversos yacimientos como el Cerro de la Gavia (Madrid), la Dehesa de la Oliva (Patones de Arriba) o Titulcia (Azcárraga 2007), por poner algunos ejemplos. Pero entre todos ellos, es El Llano de la Horca el que presenta mayor proporción de este tipo de cerámica, con más de 200 fragmentos, a los que se suman las ánforas campanas, algunos vasos de paredes finas y diversos objetos de la vajilla en bronce que aquí presentamos.

Los estudios centrados en dichos elementos de bronce específicamente tardorrepublicanos no son muy abundantes. Sin embargo, en nuestro país la vajilla de bronce genéricamente romana constituye uno de los grupos metálicos tradicionalmente más estudiados (Palol 1970; Blázquez 1976; Aurrecoechea 1991; Aurrecoechea y Fernández Ochoa 1994; Pozo 1999; etc.). La celebración de una Mesa Redonda en Lattes en 1990 (Feugère y Rolley 1991) en torno a la vajilla broncínea tardorrepublicana, supone un avance importante en el estudio de este tipo de objetos. En la Península Ibérica existen varios trabajos 
centrados en dicha vajilla, tanto en zonas concretas como la Baetica (Pozo 2000), como en determinados yacimientos (Fabião 1999; Mansel 1999; Álvarez et alii 2000; Blázquez et alii 2002). En el último trabajo de conjunto publicado en España centrado en la vajilla de esta época (Mansel 2004) llama la atención la distribución de los distintos hallazgos, destacando el vacío continuo en la zona central. Sin embargo otros trabajos posteriores que recogen cronologías más extensas amplían este panorama (Erice 2007; Aurrecoechea 2009). Con este trabajo añadimos un punto más en el mapa, que no dudamos continuará siendo completado con el avance en las investigaciones y excavaciones de la zona.

Por otro lado, un aspecto a tener en cuenta es el de los problemas de identificación de estos materiales broncíneos, derivados del carácter fragmentario de la mayoría de ellos. En muchas ocasiones, al conservarse tan solo pequeñas partes del recipiente difíciles de identificar, o elementos muy fracturados o deformados, han podido pasar desapercibidos. Esperamos que este trabajo sirva además, para facilitar su reconocimiento, sobre todo en los yacimientos del centro peninsular, donde hasta ahora eran prácticamente desconocidos.

\section{EL YACIMIENTO DE EL LLANO DE LA HORCA}

El oppidum de El Llano de la Horca, ubicado en Santorcaz (Madrid) es uno de los yacimientos más relevantes para el estudio de la Carpetania prerromana y su transición al mundo romano (Fig. 1). Ha sido objeto de numerosas campañas de excavación, desde la realizada en el año 1985 por Méndez Madariaga (sin publicar), la de 1990 llevada a cabo por Cerdeño (Cerdeño et alii 1992), hasta las ya 11 campañas realizadas por el Museo Arqueológico Regional de la Comunidad de Madrid, que proyecta la creación de un Parque Arqueológico en el lugar (Baquedano et alii 2007a; 2007b; Märtens et alii 2009; Gozalbes et alii 2011). Como una pequeña muestra del resultado de dichas excavaciones se organizó la exposición temporal de "Los últimos carpetanos. El oppidum de El Llano de la Horca" en el citado museo, abierta al público entre el 18 de abril y el 25 de noviembre de 2012 (Ruiz et alii 2012).

Las características topográficas del emplazamiento, localizado en un cerro amesetado de unas 14 hectáreas, con amplio control del territorio circundante y con accesos abruptos en algunas zonas, son las que le aportan la denominación de oppidum. Aunque, por el momento, no se ha detectado la presencia de murallas propiamente dichas, es posible que al menos

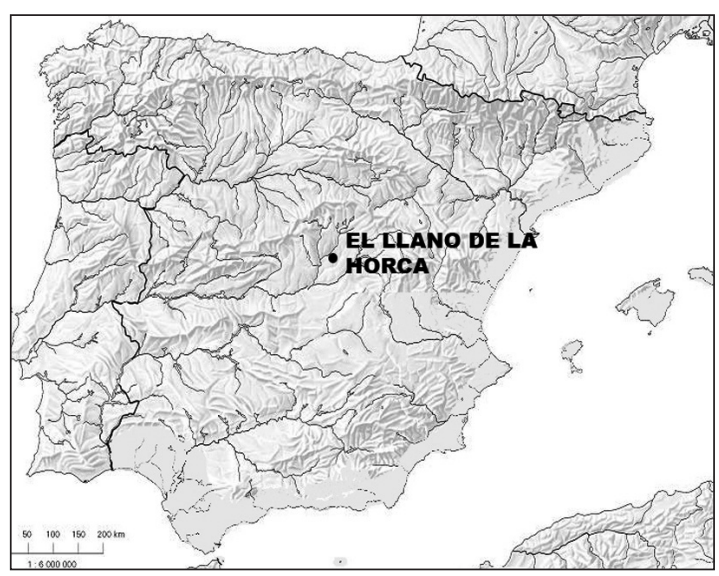

Figura 1. Localización del oppidum de El Llano de la Horca (Santorcaz, Madrid).

en la zona de acceso principal al cerro, por el este, hubiera podido existir alguna estructura de este tipo, ya que presenta una pendiente más suave y por tanto resulta de más fácil acceso (Cerdeño et alii 1992: 154; González Zamora 1999: 18). La ocupación de cerros testigo o espolones, fácilmente defendibles, con amplio control del territorio circundante y próximos a recursos fluviales, es un fenómeno que parece generalizarse en el centro de la Península Ibérica en un momento avanzado de la Segunda Edad del Hierro. Urbina propone la manifestación de este fenómeno a mediados del siglo IV a.C., fruto de un proceso interno que se produjo con anterioridad a la llegada de cartagineses y romanos (Urbina 2004: 19), aunque observa también un nuevo proceso de amurallamiento en el siglo III a.C. que relaciona con la llegada de los cartagineses, pero con un carácter mucho más parcial (2004: 20). Torres, por su parte, explica este proceso en la zona central del valle del Tajo desde el punto de vista de la mayor complejidad social que se está desarrollando y que provoca un clima de tensión generalizada que se manifiesta en los poblados fortificados (Torres 2012: 658).

En cualquier caso, la cronología propuesta para El Llano de la Horca abarca un período que se desarrolla entre mediados del siglo III a.C. o poco antes, y comienzos del siglo I a.C. o poco después (Baquedano et alii 2007a: 379). Desde el punto de vista de la numismática, en el conjunto de monedas documentadas hasta el año 2009, se observa su uso entre mediados del siglo II a.C. y el periodo sertoriano, aunque con algunas salvedades derivadas del desgaste de ciertos ejemplares de Sekaisa y con algunas pérdidas aisladas de monedas que podrían llegar a mediados del s. I a.C. (Gozalbes et alii 2011: 342-343). Por otro lado, la cronología que puede aportar el barniz negro halla- 
do en el yacimiento muestra un ámbito cronológico de recepción de estos materiales importados desde fases antiguas de su producción, tanto en el caso de la Campaniense A, como en el de la cerámica de Cales, hasta las fechas más recientes, lo que nos podría situar entre el 180 a.C. y el 40/20 a.C. Sin embargo, si tenemos en cuenta la ausencia de determinadas decoraciones en la cerámica de Cales hasta ahora no documentadas en el yacimiento, y que aparecerían a partir del 75 a.C. (Azcárraga 2011: 15; Azcárraga et alii e. p.), debemos considerar el final de la ocupación hacia esas fechas. Por tanto, son varias las vías que nos llevan a considerar una cronología general para el final de la ocupación del asentamiento en torno a las guerras sertorianas (83-72 a.C.), aunque la perduración de este tipo de materiales, tanto las monedas, como la vajilla de lujo, no nos permite realizar una afirmación categórica en este sentido.

El análisis de los bronces hallados en el yacimiento nos sitúa cronológicamente en la misma horquilla, ya que la mayoría de las producciones están en uso durante ese genérico periodo tardorrepublicano. La presencia en El Llano de la Horca de las formas más comunes y algunas poco difundidas de la vajilla de bronce de esa época colocan al yacimiento en un lugar privilegiado para la llegada de este tipo de productos a la Península, hasta ahora poco documentados en el interior de la Meseta. Contamos con dos fragmentos de asas o asas completas de distintos tipos de jarras, otra que podría pertenecer a una taza, otra de un pequeño recipiente, además de cuatro mangos o remates de cazo y tres fragmentos de coladores, lo que convierte al Llano de la Horca en uno de los yacimientos con mayor cantidad y variedad de elementos de la vajilla de bronce en contexto doméstico, solo comparable con Cabeça de Vaiamonte (Monforte), con 17 elementos documentados (Fabião 1999) y el poblado minero de la Loba (Fuenteobejuna, Córdoba), con 10 elementos (Blázquez et alii 2002).

En este sentido resulta interesante mencionar las características de los principales yacimientos peninsulares en los que se documenta este tipo de material, relacionados bien con la ocupación militar romana, bien con contextos funerarios o bien con asentamientos ibéricos, celtibéricos o del Suroeste peninsular. En el primer caso destacan los campamentos romanos de Cáceres el Viejo (Cáceres) y Renieblas (Garray, Soria), o fortalezas romanas como la de Castelo da Lousa (Mourão, Évora) y Castelo Velho do Cobres (Castro Verde, Beja). En el segundo caso, cabe destacar la necrópolis de Ampurias y en el tercero, y más numeroso, los asentamientos de Cabezo de Alcalá (Azaila, Teruel), Morro de Mezquitilla (Algarrobo, Málaga), La Loba (Fuenteobejuna, Córdoba) o Camp de les Lloses (Tona, Osona), además de los yacimientos portugueses de Moldes

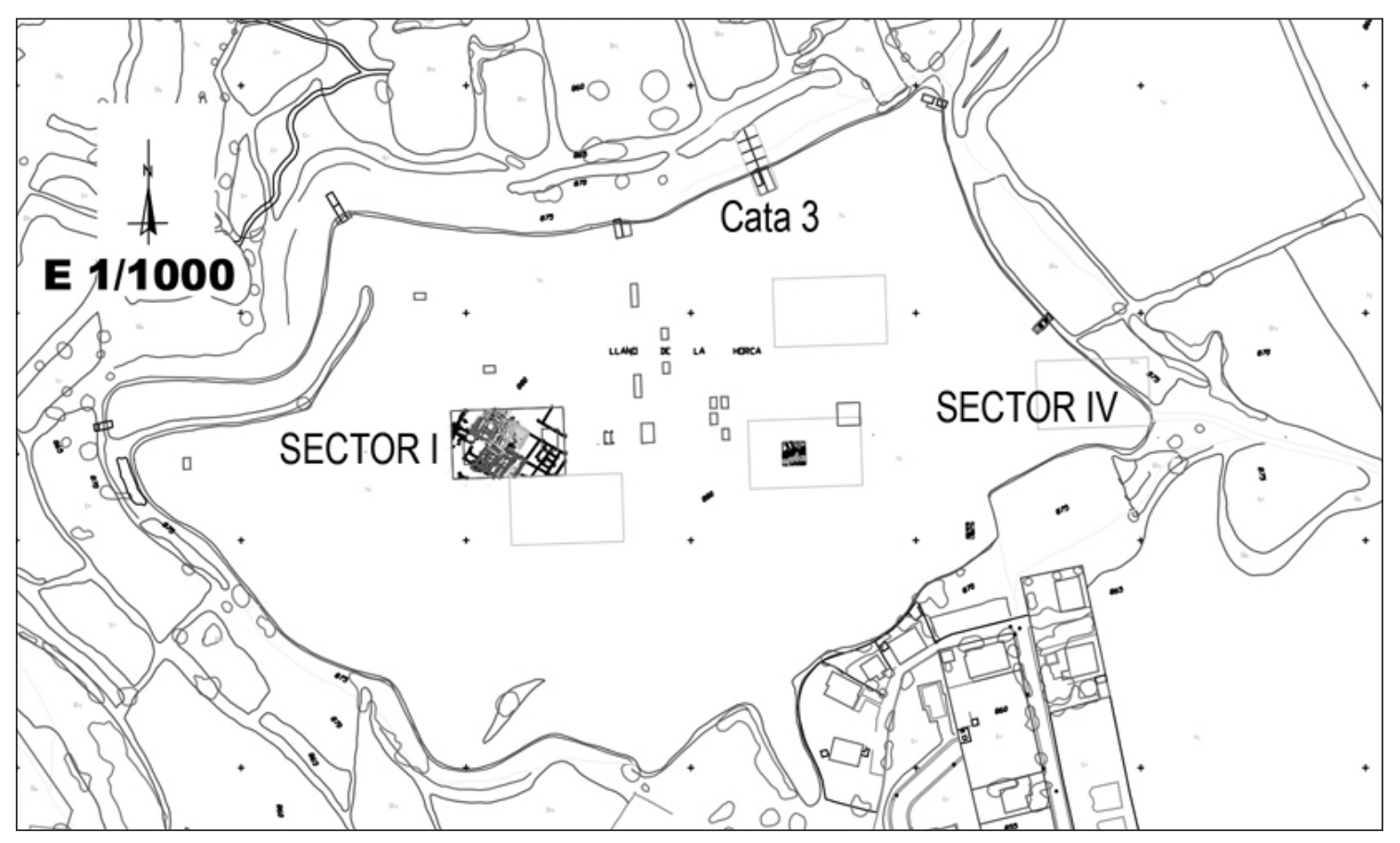

Figura 2. Plano topográfico de El Llano de la Horca, con la localización general de los hallazgos broncíneos en los Sectores I, IV y cata 3. 
(Castelo de Neiva, Viana do Castelo) y Cabeça de Viamonte (Monforte).

La muestra de vajilla de bronce analizada en este artículo recoge asas y componentes de jarras y tazas, coladores y cazos documentados en las campañas de El Llano de la Horca entre 2001 y 2012.

\section{JARRAS, TAZAS Y OTROS ELEMENTOS}

Las jarras y tazas son elementos característicos de vajilla de bronce tardorrepublicana. En el Llano de la Horca se conservan las asas de tres recipientes de distinta tipología. Estas formas completas se documentan muy rara vez en contextos domésticos, debido a su fragilidad, siendo las asas los elementos más característicos. Por otro lado, este hecho influye en la catalogación de algunos de estos objetos, ya que ciertos modelos de asas son similares en diferentes recipientes, dificultando su clasificación (Mansel 2004: 23). En nuestro caso, se conservan tres asas con un aplique parecido, un motivo de hoja cordiforme, siendo la tercera de menor tamaño y con un motivo bastante más tosco y esquemático (Fig. 3).

Los dos ejemplares de asas de mayores dimensiones se documentaron en el yacimiento durante la campaña de 2006, ambos en el interior del recinto 9 (Fig. 8), que forma parte de una de las unidades habitacionales excavadas en el Sector I del yacimiento. Se hallaron en el nivel de colmatación de dos estancias diferentes, que podrían estar relacionadas con los almacenes de la casa, ya que ambas presentan grandes contenedores y recipientes de cocina (principalmente tinajas y ollas). Además, se documentan en el contexto de la primera asa (Fig. 3.1) diversos elementos metálicos: varios clavos, una hoja de cuchillo, una vaina de puñal y un peine cardador, elementos que podrían apuntar hacia el uso de esa estancia como almacén de objetos preciados, función reforzada por algún recipiente de cerámica muy cuidada como una copa oxidante. Sin embargo, en el caso del material asociado a la segunda de las piezas (Fig. 3.2), aparte del ya mencionado material de almacenaje y cocina, llama la atención la presencia de un tonelete cerámico zoomorfo con decoración polícroma (Ruiz et alii 2012: 364 n. 239) y que quizá podría tener un carácter más simbólico. Además, aparecen piezas de vajilla fina tanto oxidante como reductora.

El ejemplar broncíneo que mejor se conserva (Fig. 3.1) presenta el motivo de hoja cordiforme completo y un apoyo para el dedo pulgar, muy desgastado pero que parece fragmentado. Esta peculiar caracte-

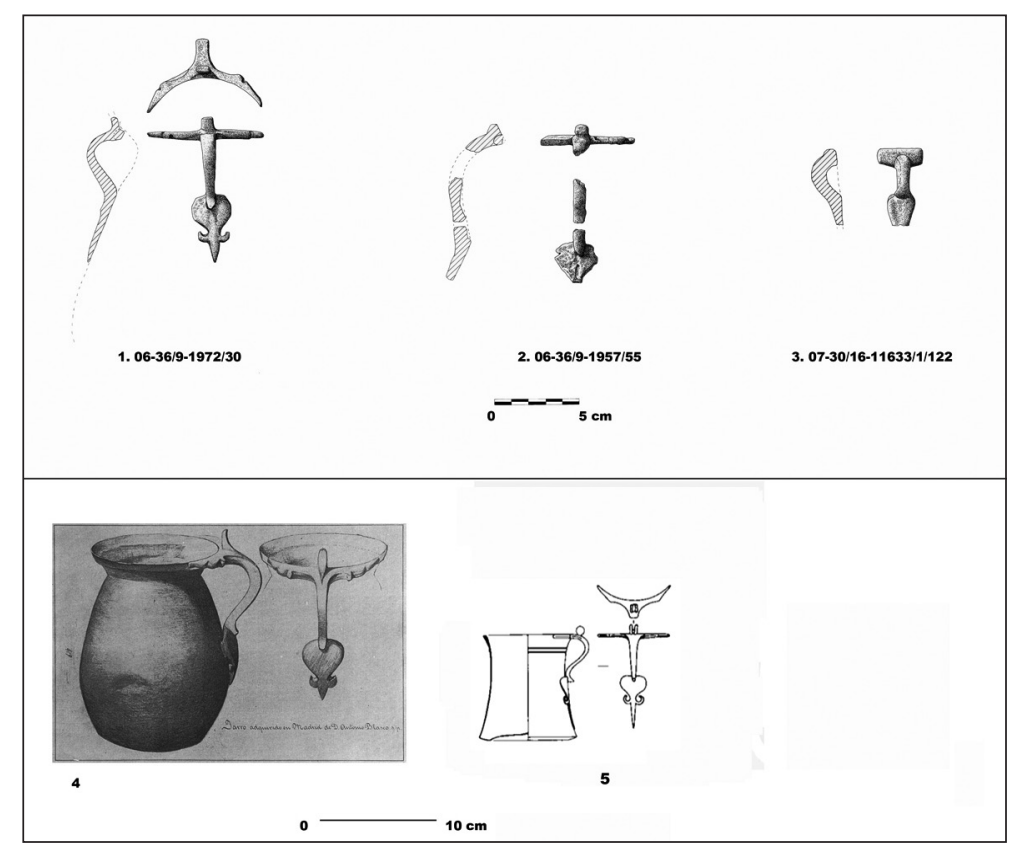

Figura 3. Jarra, taza y vaso de menor tamaño con remates de hoja cordiforme: 1,2 y 3 El Llano de la Horca (dibujos de Mayte Narváez); 4. Jarra tipo Gallarate de la colección de A. Vives (García y Bellido y García-Bellido 1993: 177); 5. Taza tipo Idria (Feugère 1991: 55, Fig. 4.1). 
rística, junto a su tamaño podrían indicar que pudo pertenecer a una jarra tipo Gallarate ${ }^{1}$ (Tizzoni 1981: 14), aunque su parecido con las tazas tipo Idria nos llevó a clasificarlo de ese modo en la catálogo de la exposición de Los últimos Carpetanos (Ruiz et alii 2012: 368 n.236).

Este tipo de jarras bicónicas, son escasas en el oeste del Mediterráneo. Aparecen ejemplares en Italia, Suiza, la antigua Yugoslavia, Hungría, Rumanía, Bulgaria, Grecia, Rusia, Alemania, Bélgica, Italia, Francia y Marruecos (Boube-Piccot 1991:30-32). Su altura oscila entre los 8,8 y los $30 \mathrm{~cm}$. (Boube-Piccot 1991: 30-32). En la Península Ibérica solo se conocen dos ejemplos, uno en Castro Sabroso (Guimãraes, Portugal) (Fabiao 1999: 169; Erice 2007: 200) y otro localizado por García y Bellido en la colección de Antonio Vives Escudero, del que no aporta procedencia (García y Bellido y García-Bellido 1993: 177 y 269) (Fig. 3.4). Por tanto, estamos ante el único ejemplar documentado con seguridad en nuestro territorio y además, contextualizado.

En cuanto a la cronología de este tipo de jarras, las más antiguas se documentan en Italia durante la primera mitad del siglo II a.C. y las más recientes en la necrópolis de San Bernardo de Ornavasso con diferentes fechas otorgadas por distintos autores, en un período que abarcaría entre el 125/100 y el 90/50 a.C. (Boube-Piccot 1991: 26). Para encontrar jarras de tipo Gallarate fuera de Italia habrá que esperar al último cuarto del siglo II a.C., siendo este para Boube-Piccot un tipo anterior al de la jarra Piatra Neamt, que se dataría entre el 125/120 y 50 a.C. en Italia y España (Boube-Piccot 1991: 26).

El segundo ejemplar (Fig. 3.2) presenta peor estado de conservación y apareció fragmentado en varios puntos. En este caso su identificación resulta aún más complicada al no conservar completo ninguno de sus remates. Basándonos en su tamaño, de en torno a $8,5 \mathrm{~cm}$. de altura, y las características formales de su remate inferior es posible que se trate de una taza tipo Idria. Este tipo de tazas tienen perfil y base cóncavas y una altura de aproximadamente $11 \mathrm{~cm}$. Su asa tendría también un pequeño apoyo para el dedo pulgar en forma de polea y el aplique de su extremo inferior representaría un motivo de hoja cordiforme (Fig. 3.5).

Las tazas de tipo Idria son un elemento característico de la vajilla de bronce, con una distribución desde la Península Itálica, Grecia y Eslovaquia, el Sur de Francia, Marruecos y la Península Ibérica (Mansel 2004: 23). En Portugal son varios los yacimientos en

\footnotetext{
${ }^{1}$ Apreciación realizada por Michel Feugère, a quien agradecemos enormemente sus observaciones.
}

los que aparece esta forma, destacando el depósito de Moldes (Fabião 1999). En nuestro territorio los hallazgos se concentran en el Suroeste, aunque el valle del Ebro y Cataluña también ofrecen ejemplos llamativos, con un total de 23 ejemplares, añadiendo el que ahora presentamos a la distribución que ofrece Erice (2007: 203). Cabe destacar el caso de la taza casi completa documentada en Tarragona, con unas medidas similares a las que podría tener el ejemplar de El Llano de la Horca (Roig 2003: 105-107).

Respecto a la cronología de este tipo de tazas especialmente interesante es la que otorga su hallazgo en el campamento republicano de Cáceres el Viejo, que aporta una fecha ante quem del 80/79 a. C. (Feugère 1991: 55). El periodo cronológico general para estas piezas se situaría entre el 120 y el 75/50 a. C. (Feugère 1991: 55), aunque para algunos autores perduraría hasta el siglo I d. C. (Roig 2003: 107), mientras que para otros los hallazgos augusteos se considerarían residuales (Erice 2007: 203).

Aparece también en El Llano de la Horca un asa de menores dimensiones y con algunas características que pueden recordar a las anteriores (Fig. 3.3), sobre todo si nos fijamos en la forma del remate inferior. Sin embargo, su reducido tamaño y la ausencia de algunos elementos característicos como el dedil, del cual no existen huellas en la pieza, nos hace pensar que se trata de una variante hasta ahora sin definir. Para esta forma solo se conoce un paralelo en la Península Ibérica, en el asentamiento de Cabeça de Vaiamonte (Monforte), donde se documentan dos ejemplares muy similares, interpretados también como variantes (Fabião 1999: 185). En nuestro caso, la longitud que conserva el asa es de 4,6 cm., aunque sería algo más larga puesto que el remate inferior está fracturado. En la representación de su sección podemos observar cómo el recipiente al que perteneciera tenía las paredes algo inclinadas pero rectas, en el punto correspondiente al asa. En el caso de las portuguesas, su longitud es algo mayor, con 5,8 y 7,4 cm., aunque al no figurar su sección no podemos comprobar su semejanza o diferencia en este aspecto, pero sí la similitud en su representación frontal (Fabião 1999: 182). Con los datos que hasta ahora poseemos podemos pensar en una variante nueva en la vajilla broncínea, aunque es algo pronto para definir sus características formales ya que aún carecemos de ejemplares más completos que permitan definirla con exactitud.

Esta pequeña asa se halló durante la campaña de 2007 en un nivel de abandono o relleno, con abundante y variado material: cerámica (de almacén, cocina o mesa), restos de fauna, elementos metálicos (clavos de hierro, botones de bronce...), alguna canica y una fusayola, estrato sobre el cual se construye parte de 
la calle Sur del Sector I (Fig. 8). Entre todo este material cabe destacar la presencia de varios elementos que nos aportan información cronológica. Por un lado, un fragmento de plato de la forma Lamb. 6 de barniz negro romano republicano perteneciente a la variante media de la producción de Cales, y por tanto con una fechas de fabricación de entre 130/120 y el 90/80 a.C. (Azcárraga et alii e. p.). Por otro lado, se documentan varias fíbulas de La Tène III que pueden fecharse en el siglo I a.C. (Ruiz et alii 2012: 324-325 n.170). Estos datos, además de ofrecer una aproximación cronológica para el asa a finales de la época republicana, resultan del mayor interés si tenemos en cuenta que se trata de un tipo broncíneo por definir.

En el yacimiento de Cabeça de Vaiamonte (Monforte), aparte de estas peculiares asas, se documentó un fragmento de asa de tipo Idria, dos de jarras de tipo Piatra Neamt y varios simpula (Fabião 1999: 182-184). Esta cantidad de elementos, junto a las características del asentamiento, hacen pensar a sus investigadores que en el lugar estuviera instalada una amplia guarnición militar romana en época tardorrepublicana, aunque no concretan más las fechas al tratarse de elementos recuperados de un expolio (Fabião 1999: 193, 185 y 186). De este modo, tanto los datos de Cabeça de Vaiamonte como los de El Llano de la Horca son muy semejantes, aunque gracias a la documentación de nuestro ejemplar, en contexto estratigráfico, se puede confirmar y precisar la cronología tardorrepublicana en torno a las últimas décadas del siglo II a.C. y las primeras del siglo I a.C.

Por último, se documenta un cuarto tipo de jarra (Fig. 4), en este caso de clara catalogación a pesar de conservarse tan solo el remate inferior decorado. Se trata del asa de una jarrita bicónica de tipo Piatra Neamt, que tendría una altura de alrededor de $11 \mathrm{~cm}$ (Fig. 4.2), conservándose tan solo su peculiar remate para el asa. En esta ocasión, se trata de un busto masculino barbado (Fig. 4.1). Se documentó en la campaña del año 2011, en un nuevo sector abierto, el Sector IV (Fig. 2), que continuó excavándose en 2012. Resulta muy interesante el contexto en el que se halla esta pieza en el yacimiento, ya que parece formar parte del entorno de lo que podría identificarse con un área de producción metalúrgica. Por tanto, cuando la pieza llega aquí, ya habría perdido su uso y sería un objeto para reciclar, junto con otros muchos. Encontramos un claro paralelo para esta actividad en el yacimiento ibérico de Camp de Les Lloses (Álvarez et alii 2000), donde aparecen también, aparte de objetos destinados a la refundición, elementos estructurales similares a los documentados en El Llano de la Horca. El Sector IV es un área del yacimiento situada en la entrada del poblado, alejada por tanto de núcleo de población principal y lugar idóneo para el desarrollo de actividades artesanales como la metalurgia o la alfarería. Aunque no se han concluido los trabajos de excavación en la zona y estas campañas están en fase de estudio, podemos destacar, que al igual que en Camp de les Lloses, aquí se ha documentado una canalización relacionada con la necesidad de agua para el trabajo de los metales y varios contenedores cerámicos con la boca cortada que podrían haber almacenado algún material necesario para este trabajo. La zona abierta por el momento en este sector no permite observar las estructuras completas, pero los resultados de una prospección geofísica realizada indican que muy próximos existen abundantes puntos de combustión o de concentración de escorias, dato también a tener en cuenta.

En cuanto a las características morfológicas de la pieza llama la atención su gran calidad. Lo más común en este tipo de elementos es la representación de rasgos lineales, como en el caso del ejemplar de Morro de Mezquitilla (Mansel 1999: 715) (Fig. 4.2), y detalles poco trabajados como en el caso de los ejemplares recientemente dados a conocer de los yacimientos conquenses de Villas Viejas y Culebras (Aurrecoechea 2009: 328 y 330 Fig. 2). En la mayoría de los casos no se aprecian con nitidez los rasgos de la cara, la barba o el peinado, tan claros en el nuestro. La plasmación de rasgos naturalistas, pone en relación este ejemplar de El Llano de la Horca con el documentado en Tossal de Polop y algo menos con los documentados en Camps de les Lloses (Tona, Osona)

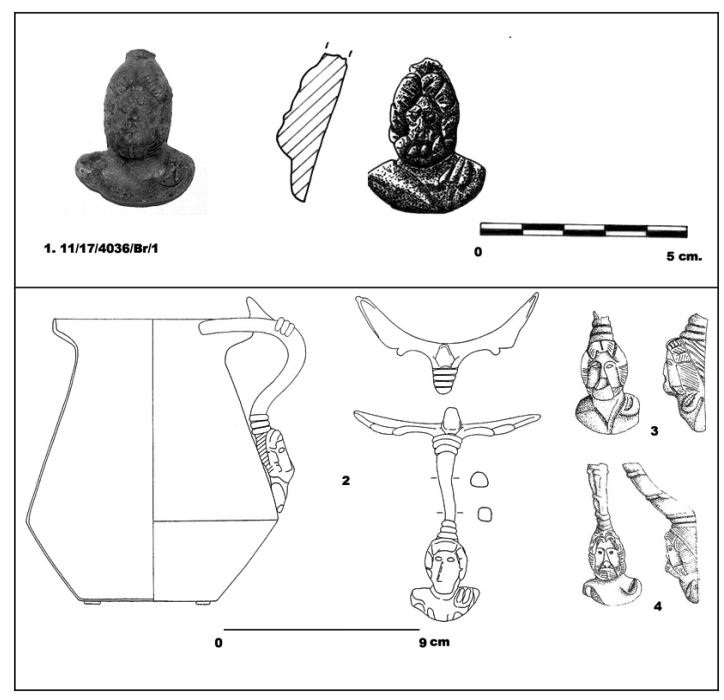

Figura 4. Jarras de tipo Piatra Neamt: 1. El Llano de la Horca (dibujos de Mayte Narváez); 2. Morro de Mezquitilla (Mansel 2000: 209); 3. Tamuda (Boube-Piccot 1988: 249) y 4. Volubilis (Boube-Piccot 1988: 251). 
(Mansel 2000: 211). Hasta el momento, la calidad de estos ejemplares era exclusiva en los localizados en la zona levantina, pero este hallazgo en el interior peninsular abre una nueva vía para el estudio de diversos aspectos, como la importancia de las rutas comerciales y los asentamientos del interior, el grado de asimilación de sus habitantes de las costumbres y gustos romanos o incluso la posible presencia de gentes itálicas en la zona.

Por otro lado, el ejemplar documentado en El Llano de la Horca presenta el extremo superior muy desgastado, lo que quizá estaría indicando la amortización de este material una vez perdido su uso como jarra. La calidad de la pieza y la representación de un busto masculino, que en ocasiones se ha relacionado con Júpiter (Boube-Piccot 1991: 25; Mansel 1999: 708; Erice 2007: 201; Aurrecoechea 2009: 328) podrían ser los motivos que llevaran a la preservación de este elemento.

Este tipo de jarra se documenta en yacimientos de Italia, el norte del Cáucaso, la costa mediterránea de Francia, Marruecos y la Península Ibérica (BoubePiccot 1991: 27 y 30). Es una forma bien representada en el ámbito peninsular, principalmente en la zona levantina y del Sur, además del valle del Ebro. Aparte de los hallazgos ya destacados, cabe señalar que en total se conocían 26 ejemplares, cifra que ha ido concretándose a través de los datos publicados por distintos autores (Mansel 2004: 24; Erice 2007: 201; Aurrecoechea 2009: 328). El ejemplar de El Llano de la Horca eleva el recuento a 27 , siendo ya el tercero documentado en la Submeseta Sur.

Por otro lado, la concentración de hallazgos en Marruecos ha sustentado la hipótesis de la existencia de un taller de asas en Tamuda (Boube-Piccot 1988:
232-233 y 239; Mansel 2000: 210-212), aunque no se han obtenido conclusiones definitivas. Boube-Piccot divide los ejemplares hallados en Marruecos en dos tipos, uno más elaborado, el tipo A, con cara barbada encuadrada por el pelo con raya en medio, la pupila de los ojos marcada y el pliegue de una clámide sobre el hombro izquierdo; y el tipo B, mucho menos trabajado, con cara estrecha y alargada, con los rasgos muy esquemáticos, lo que para Boube-Piccot podría indicar una fabricación regional (1988: 232). Atendiendo a estas clasificaciones y observando los modelos hallados en esta zona, encuadramos claramente nuestro ejemplar con el primer grupo, y destacamos el gran parecido con el del yacimiento de Volubilis (Fig. 4.4) y con otro de Tamuda (Boube-Piccot 198788: 251) (Fig. 4.3).

La cronología para las piezas peninsulares se mueve entre el 125/120 y 50 a.C., para Boube-Piccot (1991: 26), y entre el final del siglo II y el primer tercio del siglo I a.C., para Mansel (2004: 25).

\section{Coladores}

Estos útiles broncíneos se componen de una taza perforada y un asa en forma de anillo con una chapa para proteger el dedo pulgar. Forman parte también de la vajilla de bronce tardorrepublicana y se encuentran presentes en El Llano de la Horca a través de tres fragmentos pertenecientes a distintos ejemplares. Se han documentado, por el momento, un asa vertical casi completa y dos fragmentos de taza perforada, de diferentes tipologías. Una de las tazas es hemisférica (Fig. 5.3) y la otra tiene el borde exvasado con perfil carenado (Fig. 5.1), es decir, de los tipos 1 y 2 de

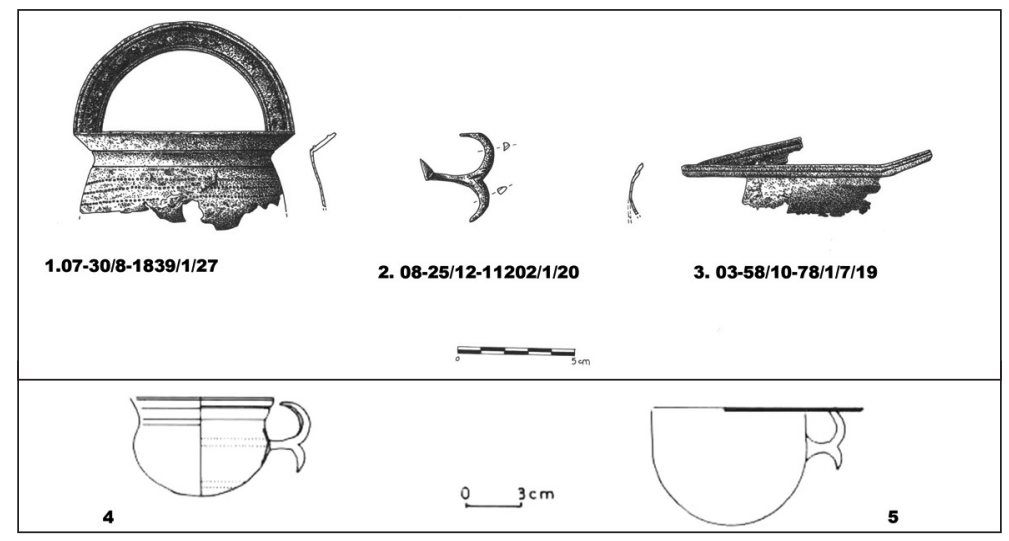

Figura 5. Coladores: 1, 2 y 3 El Llano de la Horca (dibujos de Mayte Narváez). 4 y 5. Reconstrucción de coladores con borde exvasado y hemisférico (Guillaumet 1991: 89; Fig. 1 y 3.1 ). 
Guillaumet (Fig. 5.4 y 5.5). El diámetro de estos elementos suele estar entre 7 y $13,4 \mathrm{~cm}$. En nuestro caso sólo podemos obtener esta medida del ejemplar que mejor se conserva, con $10,8 \mathrm{~cm}$. de diámetro en el borde exterior, y que destaca por su estado de conservación, muy poco frecuente al tratarse de piezas extremadamente delicadas.

La taza de colador de borde exvasado (Fig. 5.1) posee una decoración en la parte interior del borde de líneas paralelas en relieve y varias líneas incisas marcando la carena y el inicio del cuerpo. Se documentó en el 2007 en el interior del recinto 8 (Fig. 8), bajo el nivel de derrumbe de las paredes de adobes. Esta estancia se interpreta como un espacio de almacén, ya que aparecieron multitud de tinajas cerámicas. Destaca la presencia en la misma UE de un denario de la ceca de Roma acuñado en el 110109 a.C. (Gozalbes et alii 2011: 344 n2), dato que aporta una aproximación cronológica para el colador.

El asa (Fig. 5.2) salió a la luz durante la campaña de 2008, en la calle norte localizada en el Sector I (Fig. 8), formando parte de sus niveles de abandono, con abundante material cerámico en su proximidad. Un ejemplar de características muy similares se localiza en el yacimiento portugués de Mesas do Castelinho, en Almodovar, en un contexto claramente republicano (Fabião 1999: 181).

El colador de forma hemisférica y borde recto (Fig. 5.3) posee decoración incisa en la parte superior del borde, a base de líneas paralelas. Dicho ejemplar fue localizado en el interior del recinto 13B (la estancia central de una casa) (Fig. 8) formando parte del nivel de amortización de un horno ubicado en una de las esquinas y asociado a multitud de elementos de cocina como una parrilla de hierro, abundantes recipientes cerámicos y diversas herramientas como unas tijeras de esquilar.

Los coladores se documentan ampliamente en Europa central, sur de Francia y Marruecos (Guillaumet 1991: 93-95). En la Península Ibérica son variados los hallazgos de este tipo, que se concentran en el suroeste, Lusitania y la costa levantina, siendo los ejemplares de El Llano de la Horca los primeros documentados la zona carpetana. En el área portuguesa aparecen hasta en 10 yacimientos diferentes, entre ellos en contextos inequívocamente republicanos destacan los hallazgos de Serra de Segóvia, Pedrão y Mesas do Castelinho (Fabião 1999: 180). En nuestro territorio algunos ejemplos característicos son los de La Alcudia, la Colonia Celsa o El Raso de Candeleda, entre otros (Erice 2007: 199-200). El elemento que se conserva en la mayoría de los casos conocidos es el asa, lo que hace más llamativo el caso de El Llano de la Horca con la presencia de dos tazas de colador.
El ámbito cronológico genérico de este útil broncíneo abarca desde finales del siglo II a.C. hasta época tiberiana (Guillaumet 1991: 92). En la Península la cronología más precisa la aporta el ejemplar del campamento romano de Cáceres el Viejo, como hemos ya señalado anterior al 80/79 a.C., ya que fue destruido durante la guerra contra Sertorio (Ulbert 1984: 202205; Abásolo et alii 2008: 118).

\section{Cazos}

Los cazos que forman parte de la vajilla de bronce tardorrepublicana se dividen en dos tipos, los de mango horizontal, muy comunes, y los de mango vertical, menos abundantes en la Península Ibérica. En El Llano de la Horca han aparecido, por el momento, tres fragmentos del primer tipo y uno del segundo.

En el primer grupo encontramos un fragmento de la parte inferior del mango que conserva parte de los brazos que sujetarían el cazo (recipiente que constituiría un elemento separado del mismo y que no se conserva), además de dos fragmentos del remate del mango terminados en cabeza de cánido o de ave. El primer fragmento (Fig. 6.1) se halló en el nivel superficial del porche de una de las casas, que abre a la calle norte (Fig. 8). El segundo fragmento (Fig. 6.2) apareció en el nivel vegetal del yacimiento y por tanto de nuevo sin contexto. El tercero (Fig. 6.3) se encontró en una estancia de almacén del recinto 3 (Fig. 8), entre el derrumbe de la pared de adobes, junto a abundantes restos cerámicos y metálicos. Entre los primeros aparecen tanto tinajas de almacén y factura tosca, como producciones más cuidadas (tinajillas o cuencos oxidantes). Entre los objetos metálicos destaca la presencia de una placa de cinturón de bronce, de tipo celtibérico y decorada (Ruiz et alii 2012: 326 n. 72), un brazalete de bronce (Ruiz et alii 2012: 327 n. 73), una fíbula de La Tène IV que puede fecharse en el siglo I a.C. (Ruiz et alii 2012: 324-325 n. 70) y dos monedas de cronología tardorrepublicana: un semis de imitación de la ceca de Roma y un denario de la ceca de Sekobirikes (Gozalbes et alii 2011: 344 n. 5 y 348 n. 33).

En cuanto a la tipología del primer fragmento se trata de un mango ternario, es decir del tipo A de Castoldi (1991: 64-65) con una parte en forma de remo y otra intermedia en forma de maza, estando perdida la tercera que también tendría forma de remo. Los remates de cazo conservados podrían adscribirse o bien a mangos ternarios del tipo A o binarios del tipo B, únicamente con dos partes, una en forma de maza y otra de remo (Castoldi 1991: 64-66). Su longitud suele oscilar entre los 27 y los $35 \mathrm{~cm}$. Ambos son tipos bien 


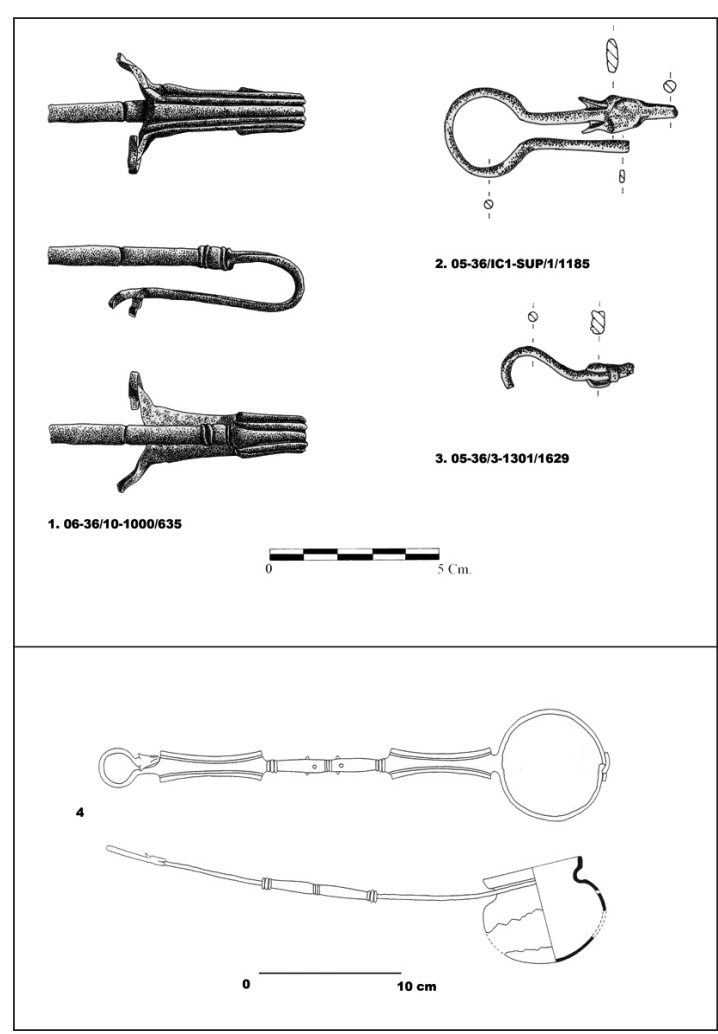

Figura 6. Cazos de mango horizontal: 1, 2 y 3 El Llano de la Horca (dibujos de Mayte Narváez); 4. Cazo ternario de La Custodia (Viana, Navarra), (Mansel 2000: 201).

representados en la Península Ibérica, sobre todo en el sur y suroeste, donde destacan, por ejemplo, el ejemplar ternario documentado en Morro de Mezquitilla (Mansel 1999: 709 y 713, 2000: 199), el de Capote (Berrocal 1994: 273-274) o el de La Custodia (Mansel 2000: 201 y 220) (Fig. 6.4), habiéndose documentado hasta 17 hallazgos de esta variante (Erice 2007: 198). En el centro peninsular contamos únicamente con los ejemplares hallados en Arganda (Madrid) y en Villas Viejas (Cuenca) (Aurrecoechea 2009: 339 y 330. Fig. 2). Mangos binarios se conocían hasta ahora 12 ejemplares procedentes de los yacimientos de Azaila, Lacipo-Casáres, Castellares, Sevilla y Bombarral, Lomba do Canho, Cabeça de Vaiamonte y Mesas do Castelinho (Erice 2007: 198). Fuera de la Península se documentan también desde Italia central y del norte al sur de Francia (Mansel 2004: 20).

En lo referente a la cronología de estas piezas en la Península Ibérica, aparecen en el primer tercio del siglo I a.C., de nuevo en el campamento de Cáceres el Viejo, y en Morro de Mezquitilla entre la segunda mitad del siglo II a.C. y la primera del I a.C. (Mansel 2004: 20).

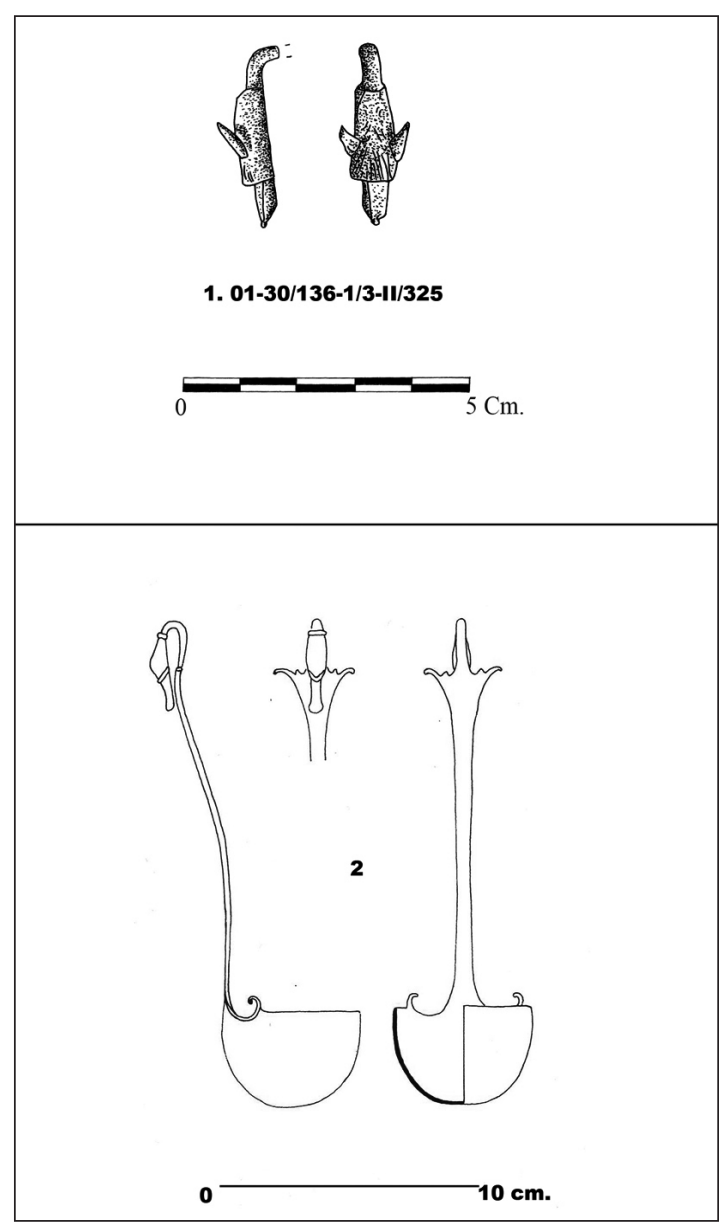

Figura 7. Cazos de mango vertical: 1. El Llano de la Horca (dibujo de Mayte Narváez); 2. Modelo de simpulum de tipo helenístico (Martín 1990: 160; Fig. 5).

Para terminar con los cazos resta analizar el fragmento de simpulum vertical documentado en El Llano de la Horca. En este tipo de elementos el recipiente hemisférico y el mango vertical conforman una única pieza (Fig. 7.2). Se conserva un pequeño fragmento (Fig. 7.1) que se corresponde con el remate en forma de cabeza de cánido, muy bien trabajada, con detalles como las incisiones que marcan el pelaje en la parte superior. Salió a la luz en la campaña del año 2001, en uno de los sondeos realizados en un extremo de la meseta del cerro, al noroeste, con el objeto de localizar posibles restos de muralla. El contexto arqueológico es el del derrumbe de un recinto habitacional, sin que se pueda precisar la estancia concreta de la casa al haberse abierto tan solo una superficie de $3 \times 9$ m. Junto a este remate de cazo se encontraron otros elementos destacados como un fragmento de aguja de hueso con decoración en espiral y otro cerámico que 
podría corresponderse con una pre-sigillata oriental que, de ser así, se dataría en torno a mediados del siglo I a.C.

Se constata una amplia difusión de estos simpula en Italia, Francia y el territorio de la antigua Yugoslavia. Aunque su presencia en la Península Ibérica es relativamente escasa, destaca el ejemplar portugués atribuido a la villa romana de Torre de Palma, que no conserva el remate (Fabião 1999: 177 y 179), los de Azaila (Beltrán Lloris 1976: 169), los de Cáceres el Viejo (Ulbert 1984: 219 y 220) o el hallado en las excavaciones de la Catedral de Pamplona y recientemente publicado (Erice 2012), entre algunos otros (Erice 2007: 199). Estos simpula son habituales en el mundo helenístico-romano, tanto los decorados con cabeza de cánido como de cisne o pato (Feugère 1991: 72), y se consideran provenientes probablemente de la Campania (Werner 1954), con un origen etrusco evolucionado del modelo griego (Feugère 1991: 72). En cuanto a su cronología en la Península, se extienden desde finales del siglo v o principios del IV a.C. y las guerras sertorianas (Martín Valls 1990: 157) sin que se aprecie ningún tipo de evolución estilística en este periodo. Fuera de la Península Ibérica se documentan hasta mediados del siglo I a.C.

\section{CONSIDERACIONES FINALES}

Los elementos aquí presentados, pertenecientes a la vajilla de bronce tardorrepublicana recuperada en El Llano de la Horca, permiten avanzar en el conocimiento de este tipo de piezas en el centro peninsular. Aumentan significativamente la variedad y cantidad de dichos elementos hallados hasta ahora en esta zona. La temprana influencia itálica en el uso de este tipo de vajillas se constataba sobre todo en la zona levantina y el sur de la Península. Este conjunto permite no solo avanzar significativamente en el conocimiento de los procesos de romanización de El Llano, sino que lo pone en relación con yacimientos de las mismas características, que tradicionalmente se han considerado con una más temprana asimilación de las costumbres romanas. El estudio conjunto de todos ellos permitirá avanzar en el conocimiento del origen, difusión y uso de la vajilla broncínea en poblados de época tardorrepublicana en Hispania.

La funcionalidad que se atribuye de manera general a estas vasijas de bronce es la del servicio para beber vino, aunque existen otras hipótesis tradicionales como la de formar parte del servicio de baño, para las abluciones antes o durante el banquete o incluso un uso en la cocina (Bolla 1991: 143). Siguiendo la hipótesis más generalizada, las jarritas y las tazas servirían para contener o consumir el vino, los cazos se utilizarían para servir o medir, y en el caso de los de mango horizontal, al ser éste largo y móvil, podrían usarse para calentar agua con especias añadidas, que se mezclarían con el vino (Mansel 2004: 27). Los coladores, por su parte, solo pudieron usarse para filtrar las bebidas. En el caso de los simpula de tipo helenístico, se les suele relacionar con un uso ritual, ya que aparecen representados en los denarios de la República romana como instrumentos sagrados (Crawford 1974; Martín 1990: 159). Respecto a esto hay que señalar que ambos tipos de cazos, no solo los de tipo helenístico, forman parte de los ajuares de las necrópolis (Martín 1990: 161) y, como hemos visto en El Llano de la Horca, los encontramos conjuntamente también en contextos domésticos. Con lo cual, un mismo objeto pudo tener variados usos, tanto cotidianos como rituales, aunque en esos usos el vino fuera el protagonista, bien para consumo o para libaciones rituales.

Respecto al origen de estas piezas, se consideran generalmente itálicas, ya que circularon junto con el vino de la misma procedencia contenido en las ánforas Dressel 1, los vasos de paredes finas, además de la vajilla de mesa de barniz negro (Castoldi 1994: 117), elementos todos presentes en el yacimiento de El Llano de la Horca (Ruiz et alii 2012). También es probable que estos elementos broncíneos llegaran a fabricarse o imitarse en nuestro territorio o en otros, como así ocurre con la vajilla de barniz negro o las paredes finas. En el caso concreto de la vajilla de bronce recordemos la hipótesis acerca de la existencia de un taller de asas de tipo Piatra Neamt en Tamuda (Boube-Piccot 1987-88: 232-233 y 239), a la que habría que añadir la más improbable variante hispana de cazos con mango horizontal en forma de varilla lisa, por el momento solo documentada en la Península Ibérica (Mansel 2004: 27). En el caso de los coladores se considera que el sur de Italia es el lugar de producción principal para este tipo de elementos (Guillaumet 1991: 92) y la Campania podría ser concretamente la zona de producción de los cazos de mango vertical. La presencia de estos objetos de bronce, junto a otros cerámicos como ánforas o paredes finas, muestra a las claras la asimilación del consumo del vino. Esta temprana influencia itálica se suele asociar con la conquista romana y los movimientos de tropas, ya que fueron también objetos transportados por los ejércitos republicanos sobre todo en la transición entre el siglo II y I a.C. (Berrocal 1994: 274).

En El Llano de la Horca se conservan restos de casi todos los objetos que componen el servicio de bebida en bronce. Algunos elementos no documentados por 


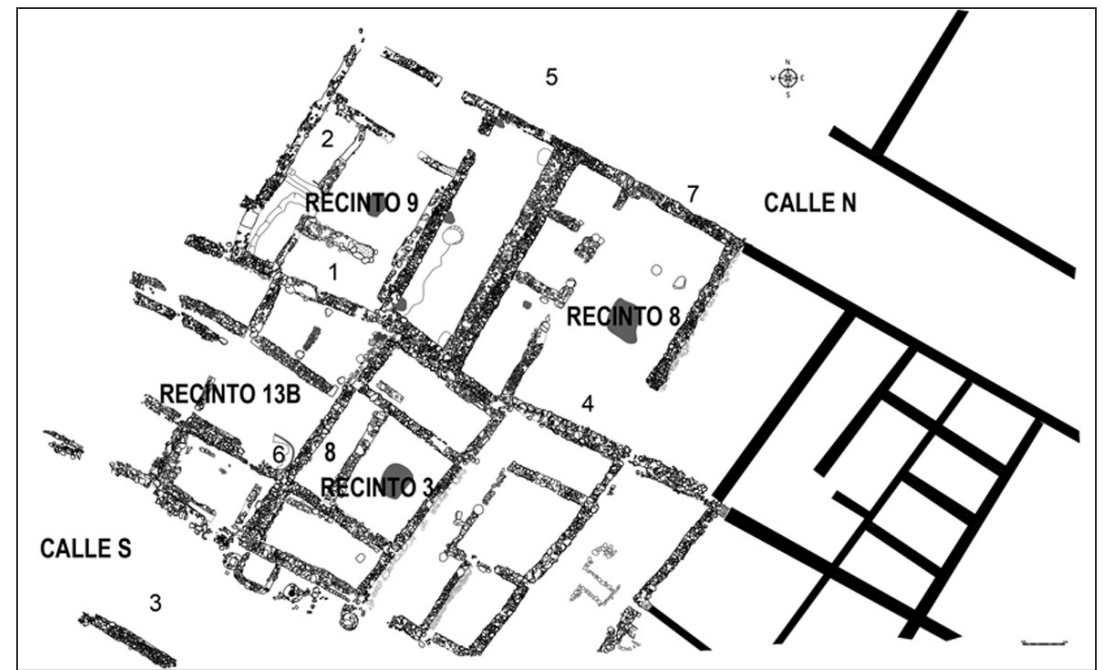

Figura 8. Detalle de la distribución de los hallazgos en el Sector I: 1. Asa de jarra tipo Gallarate, 2. Asa de taza tipo Idria, 3. Asa de variante de forma desconocida, 4. Colador de borde exvasado, 5. Asa de colador, 6. Fragmento de colador hemisférico, 7. Fragmento de mango de cazo tipo A, 8. Fragmento de remate de cazo.

el momento y pertenecientes también a la vajilla de bronce tardorrepublicana serían las ánforas de tipo Adge o la sartén baja. La experiencia nos demuestra que cuanto más se excava y estudian los materiales, más formas diferentes documentamos, tanto en la vajilla cerámica como en la broncínea, por lo que nos parece arriesgado hablar de un servicio básico de bronce en este yacimiento. Sin embargo, llama la atención que los elementos se repiten, es decir, se hallan representados en su mayoría por varios ejemplares de cada tipo, y en diferentes casas o zonas del yacimiento, lo cual podría estar indicando un uso de este tipo de vajilla más generalizado del que podría pensarse (Fig. 2 y 8).

En cuanto a la cronología que pueden aportar estar piezas de bronce en El Llano de la Horca, no nos permite precisar más las fechas de abandono del yacimiento, ya que la gran mayoría se documentan hasta mediados del siglo i a.C. Destaca la presencia de muchos de estos elementos en contextos sertorianos, aunque no implicaría que más allá de esas fechas dejaran de usarse.

En resumen, la excepcionalidad de la existencia de estos elementos de vajilla en El Llano de la Horca no lo es solo por su hallazgo en el centro de la Península Ibérica, sino también por la considerable cantidad y variedad documentada en un único yacimiento, que significativamente no es un entorno funerario. Con dos asas de jarras, una taza, un pequeño recipiente por cuatro fragmentos de cazos y tres de coladores, el conjunto broncíneo de El Llano de la Horca se convierte, con 11 ejemplares, en uno de los más importantes de la Península.

\section{BIBLIOGRAFÍA}

Abásolo Álvarez, J. A., González Fernández, Mª L. y Mora Serrano, B. 2008: "Recientes investigaciones en el Campamento de Cáceres el Viejo", P. J. Sanabria Marcos (ed.), Arqueología urbana en Cáceres. Investigaciones e intervenciones recientes en la ciudad de Cáceres y su entorno. Actas de las Jornadas de Arqueología del Museo de Cáceres, Cáceres, 115-143.

Álvarez Arza R., Durán i Caixal, M., Mestres Santacreu, I., Molas Font, M. D. y Principal Ponce, J. 2000: "El jaciment del Camp de les Lloses (Tona, Osona), i el seu taller de metalls", III Reunió sobre Economia en el Món Ibèric, Saguntum, Extra 3, Valencia, 271- 281.

Aurrecoechea Fernández, J. 1991: "Vajilla metálica de época romana en los museos de Ciudad Real, Jaén y Linares", Espacio, Tiempo y Forma, Serie II, Historia Antigua IV, Madrid, 223-254.

Aurrecoechea Fernández, J. 2009: "Vajilla metálica de época romana en la región central de Hispania (actuales CCAA de Madrid y Castilla-La Mancha)", Sautuola XV, 327-348.

Aurrecoechea Fernández, J. y Fernández Ochoa, C. 1994: "Vajilla metálica de época romana en el Museo Arqueológico provincial de Oviedo", Bo- 
letín del Real Instituto de Estudios Asturianos 144, 687-694.

Azcárraga Cámara, S. 2007: "El inicio de la romanización en la región madrileña: nuevas perspectivas para la investigación”, A. Dávila (ed.) Estudios sobre la Edad del Hierro en la Carpetania, Zona Arqueológica 10, I, Madrid, 322-341.

Azcárraga Cámara, S. 2011: "La cerámica de barniz negro de el Llano de la Horca (Santorcaz)". Informe inédito.

Azcárraga Cámara, S., Märtens Alfaro, G., Contreras, M., Ruiz Zapatero, G. y Baquedano, E. e. p.: "La cerámica republicana de barniz negro en El Llano de la Horca (Santorcaz, Madrid)", I Simposio sobre los Carpetanos: Arqueología e Historia de un pueblo de la Edad del Hierro, Alcalá de Henares, en prensa.

Baquedano, E., Contreras, M., Märtens, G. y Ruiz Zapatero, G. 2007a: "El oppidum carpetano de El Llano de la Horca (Santorcaz, Madrid)", A. Dávila (ed.) Estudios sobre la Edad del Hierro en la Carpetania, Zona Arqueológica 10, II, Madrid, 374-394.

Baquedano, E., Contreras, M., Märtens, G. y Ruiz Zapatero, G. 2007b: "En busca de los últimos carpetanos. El poblado de El Llano de la Horca (Santorcaz, Madrid)", Madrid Histórico 11, 8-17.

Beltrán Lloris, M. 1976: Arqueología e Historia de las ciudades antiguas del Cabezo de Alcalá de Azaila (Teruel), Zaragoza.

Berrocal-Rangel, L. 1994: "La falcata de Capote y su contexto. Aportaciones a la fase tardía de la cultura céltico-lusitana", Madrider Mitteilungen 35, 258-291.

Blázquez, J. M. 1976: “Antigüedades íberas, romanas y visigodas del Museo Lázaro Galdiano”, Goya 131, 284-285.

Blázquez, J. M. Domergue, C. y Sillieres, P. 2002: "La Loba (Fuenteobejuna, Córdoba)", La Mine et le village minier antiques, Bourchaux.

Bolla, M. 1991: "Considerazioni sulla funzione dei vasi in bronzo tardorrepubblicani in Italia septentrionale", M. Feugère y C. Rolley (eds.), La vaisselle tardo-républicaine en bronze. Actes de la table ronde du CNRS, Publications du Centre des recherches sur techniques gréco-romaines 13, Dijon, 143-153.

Boube-Piccot, C. 1987-88: "Anses de cruches bitronconiques à carène basse (type italique de la première moité du premier siècle avant J. C.) découvertes au Maroc", Bulletin d'archéologie marocaine 17, 231-262.

Boube-Piccot, C. 1991: "Les cruches", M. Feugère y C. Rolley (eds.), La vaisselle tardo-républicaine en bronze. Actes de la table ronde du CNRS, Publications du Centre des recherches sur techniques gréco-romaines 13, Dijon, 23-45.

Castoldi, M. 1991: "Simpula a due pezzi a manico orizontale", M. Feugère y C. Rolley (eds.), La vaisselle tardo-républicaine en bronze. Actes de la table ronde du CNRS Publications du Centre des recherches sur techniques gréco-romaines, 13, Dijon, 63-72.

Castoldi, M. 1994: "Recipienti in bronzo d'età Romana provenienti da Milano", Akten der 10 Internationalen Tagung über antike Bronzen, Forschungen und Berichte zur Vor- und Frühgeschichte in Baden-Württemberg 45, Stuttgart, 117-127.

Cerdeño, Ma L., Martín, E., Marcos, F. y Ortega, J. 1992: "El yacimiento prerromano de Santorcaz (Madrid)", Arqueología, Paleontología y Etnografía 3, 132-170.

Crawford, M. H. 1974: Roman Repubican coinage, I-II, Cambridge.

Erice Lacabe, R. 2007: "La vajilla de bronce en Hispania”, C. Fernández Ibáñez (ed.), Metalisteria de la Hispania Romana, Sautuola XIII, 197-215.

Erice Lacabe, R. 2012: "Un mango de simpulum procedente de las excavaciones de la catedral de Pamplona", Trabajos de Arqueología Navarra 24, 143-148.

Fabião, C. 1999: “A propósito do depósito de Moldes, Castelo de Neiva, Viana do Castelo: a baixela romana tardo-republicana em bronze no extremo ocidente peninsular", Revista Portuguesa de Arqueología 2, 1, 163-198.

Feugère, M. 1991: "Les simpulums à manche vertical”, M. Feugère y C. Rolley (eds.), La vaisselle tardo-républicaine en bronze. Actes de la table ronde du CNRS, Publications du Centre des recherches sur techniques gréco-romaines 13, Dijon, 72-87.

Feugère, M. y Rolley, C. 1991: La vaisselle tardorépublicaine en bronze. Actes de la table ronde du CNRS, Publications du Centre des recherches sur les techniques gréco-romaines 13, Dijon.

García y Bellido, A. y García-Bellido, M. P. 1993: Álbum de dibujos de la colección de bronces antiguos de Antonio vives Escudero, Anejos de Archivo Español de Arqueología XIII, Madrid.

González Zamora, C. 1999: Fíbulas en la Carpetania, Madrid.

Gozalbes, M., Märtens, G., Contreras, M., Baquedano, E. y Ruiz-Zapatero, G. 2011: "Dinero en Carpetania: hallazgos monetarios en El Llano de la Horca (Santorcaz, Madrid)", Ma. P. García-Bellido, L. Callegarin y A. Jiménez Díez (eds.), Barter, Money and coinage in the ancient Mediterranean 
(10th- 1st century BC), Anejos Archivo Español de Arqueología LVIII, Madrid, 335-352.

Guillaumet, J. P. 1991: "Les passoires", M. Feugère y C. Rolley (eds.), La vaisselle tardo-républicaine en bronze. Actes de la table ronde du CNRS, Publications du Centre des recherches sur techniques gréco-romaines 13, Dijon, 89-95.

Mansel, K. 1999: "Vasijas de bronce tardorrepublicanas del Morro de Mezquitilla (Algarrobo, Málaga)", XXIV Congreso Nacional de Arqueología, IV, Romanización y desarrollo urbano en la Hispania republicana, Murcia, 707-717.

Mansel, K. 2000: "Spätrepublikanisches Bronzegeschirraus aus der Siedlung vom Morro de Mezquitilla (Algarrobo, Málaga)", Madrider Mitteilungen 41, 197-225.

Mansel, K. 2004: "Vajilla de bronce en la Hispania republicana”, R. Olmos y P. Rouillard (coord.), La vajilla ibérica en época helenística (siglos IV - III a. C. al cambio de era), Collection de la Casa de Velázquez 89, Madrid, 19-30.

Märtens Alfaro, G., Contreras Martínez, M., Ruiz Zapatero, G y Baquedano Pérez E. 2009: "El Llano de la Horca (Santorcaz). Un yacimiento carpetano en los albores de la romanización", Actas de las Terceras Jornadas de Patrimonio Arqueológico en la Comunidad de Madrid, Madrid, 201-232.

Martín Valls, R. 1990: "Los simpula celtibéricos", Boletín del Seminario de Estudios de Arte y Arqueología 56, 144-169.

Palol, P. de 1970: "Necrópolis hispanorromanas del siglo IV en el Valle del Duero. III. Los vasos y recipientes de bronce", Boletín del Seminario de Estudios de Arte y Arqueología 36, 222- 223.
Pozo, S. F. 1999: “La vajilla broncínea romana en el Museo Histórico Municipal de Priego de Córdoba", Antiquitas 9, 45-54.

Pozo, S. F. 2000: "La vajilla metálica -bronce y platatardorrepublicana en la provincia Baetica", Antike Bronceen, Werkstattkreise: Figuren und Geräte, Akten des 14. Internationalen Kongresses für antike Bronzen, Kölner Jahrbuch 33, Köln, 417-434.

Roig, J. F. 2003: "El vasos de bronze del pou Cartanyà. Un aixovar sacre de l'antiga Tarraco", Revista d'Arqueologia de Ponent 13, 83-124.

Ruiz Zapatero, G., Märtens Alfaro, G., Contreras Martínez, M., Baquedano, E. 2012: Los últimos Carpetanos. El oppidum de El Llano de la Horca (Santorcaz, Madrid). Catálogo de la exposición del Museo Arqueológico Regional de la Comunidad de Madrid, 18 Abril-25 noviembre 2012, Madrid.

Tizzoni, M. 1981: "La cultura tardo La Tène in Lombardia", Studi Archeologici 1, 3-40.

Torres Rodríguez, J. de 2012: La tierra sin límites. Territorio, sociedad e identidades en el valle medio del Tajo (s. IX-I a. C.), Tesis Doctoral, Universidad Complutense de Madrid.

Ulbert, G. 1984: Cáceres el Viejo. Ein späterepublickanisches Legionslager in Spanisch-Extremadura, Madrider Beiträge 11, Mainz.

Urbina, D. 2004: "Claves de la secuencia del poblamiento de la Edad del Hierro en el Centro de la Península", Actas do IV Congresso de arqueología peninsular. As idades do Bronce e do Ferro na Península Ibérica, Faro, 15- 27.

Werner, J. 1954: "Die Bronzekanne von Kelheim", Bayerische Vorgeschichtsblätter 20, 43-73.

Recibido: 25-03-2013

Aceptado: 03-09-2013 